\title{
Plasma Biomarker for Alzheimer's Disease: Are We Ready Now for Clinical Practice and Drug Trials?
}

\section{A. Nakamura}

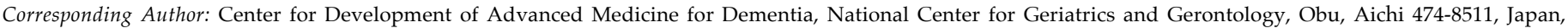
nakamura@ncgg.go.jp

7 o facilitate disease-modifying clinical trials for Alzheimer's Disease (AD), a bloodbased amyloid- $\beta(\mathrm{A} \beta)$ biomarker, which can accurately detect an early pathological signature of $\mathrm{AD}$ at prodromal or preclinical stages, has been strongly desired, because it is simpler, less invasive and less costly compared to PET or lumbar puncture. Despite plasma $\mathrm{A} \beta$ biomarkers having been extensively investigated, most studies failed to demonstrate clinical utility $(1,2)$, and at the end of 2016, there was a rather pessimistic mood that this objective might be impossible to realize (3). However, since the latter half of 2017, the situation appears to have changed dramatically, in that several groups have reported potential clinical utility of plasma A $\beta$ biomarkers using different methodologies (4-7). Especially, immunoprecipitation followed by mass spectrometry (IP-MS) assays have shown promising converging evidence. In 2014, we, the National Center for Geriatrics and Gerontology (NCGG) and Koichi Tanaka Mass Spectrometry Research Laboratory at Shimadzu Corporation (Shimadzu), reported that the plasma ratio of A $31-42$ to a novel APP669-711 fragment (APP669-711/ $A \beta 1-42)$ as determined by IP-MS could discriminate high $\mathrm{A} \beta(\mathrm{A} \beta+)$ individuals from low $\mathrm{A} \beta(\mathrm{A} \beta-)$ individuals (classified using PiB-PET) with more than $90 \%$ accuracy $(\mathrm{n}=62)$ (8). In 2017, the Washington University group analyzed detailed kinetics of plasma $A \beta s$, and reported that $A \beta 42 / A \beta 40$ as measured by IP-MS could distinguish $\mathrm{A} \beta+$ and $\mathrm{A} \beta$ - individuals with $88.7 \%$ areas under the curve value $(n=41)(5)$. Then very recently, we, in collaboration with the Australian Imaging, Biomarker and Lifestyle Study of Aging (AIBL), have demonstrated that plasma biomarkers, APP669-711/A $\beta 1-42, A \beta 1-40 /$ $\mathrm{A} \beta 1-42$, and their composites (composite biomarker), as generated by improved IP-MS methodology performs very well in larger independent datasets: a discovery dataset (NCGG, $\mathrm{n}=121$ ) and a validation dataset (AIBL, $\mathrm{n}=252$ which includes $\mathrm{n}=111$ PiB-PET and 141 with other ligands) both of which included individuals with normal cognition, MCI and AD. Particularly, the composite biomarker showed very high AUCs in both datasets (discovery $96.7 \%, \mathrm{n}=121$, and validation $94.1 \%, \mathrm{n}=111$ ) with accuracy c.a. $90 \%$ when using PiB-PET as standard of truth. The findings of the study were considered to be robust, reproducible and reliable because biomarker performance was validated in a blinded manner using independent data sets (Japan and Australia) and involved an established large-scale multicenter cohort (AIBL).

We consider the plasma biomarkers to be potentially useful for the following applications: 1) Drug trials: used as a prescreening tool before PET scans to recruit prodromal/preclinical AD individuals efficiently. In addition, they may be also useful as monitoring tools for interventions, although we have as yet no data. 2) Daily clinical practice: as there is usually some degree of diagnostic uncertainty about a clinical diagnosis of AD, the plasma biomarkers are expected to be helpful for the differential diagnosis and aid in determining therapeutic strategies, by providing information for individual status of brain $A \beta$ amyloid accumulation. 3) Medical checkup for general elders: in the future, the plasma biomarkers may be used as a medical checkup tool to screen individuals at risk for AD. However, we should be very careful for this application, because the information of "having AD risk" can be too impactful for the general population. Thus, the application should be coupled with developments of effective drugs or effective preventive interventions. Social and ethical agreements will be needed to handle such information, so that individuals having AD risk are not treated unfairly. However, if all of these concerns are solved, the plasma biomarkers may contribute to early intervention or risk management of $\mathrm{AD}$ that could lead to great reduction of the cost of dementia therapeutic strategies across the world.

Of course, there are still a lot of issues that need to be addressed before general clinical application, as discussed in our paper (7). First, further validation studies involving different large-scale cohorts coupled with longitudinal data will be needed. Second, standardized operating procedures (SOP) for the analytical process as well as the pre- and post-analytical steps should be established (9), preferably through an international consortium. Under the controlled SOP, optimal common cut-off values as well as the optimal mathematical generation of the composite biomarker should be established. Third, in clinical trials targeting $A \beta$ reduction, the usefulness of this plasma $A \beta$ biomarker as a monitoring tool remains to be evaluated. Additionally, 
biomarker performances for the differential diagnosis of other types of dementia need to be established.

To fulfil all these requirements, it may take more than five years, especially because the second issue is very demanding and difficult to solve, even for CSF biomarkers. However, if we restrict the use of our plasma biomarker as a "prescreening tool before PET scans" for drug trials, we expect it may be possible to start initial application rather soon, presumably within a year. Assume when recruiting preclinical $\mathrm{AD}$ individuals from general elderly population aged around 75 years, of which prevalence of $\mathrm{A} \beta$-positive population is about $30 \%$ (10), there would be $70 \%$ negative scans for A $\beta$-PET imaging. However, according to our estimation (7), prescreening by the plasma biomarker potentially reduce the number of negative PET scans to about $1 / 7$ or even less. This can significantly reduce the costs for drug trials and improve the efficiency for recruitment. For the prescreening application, we consider that it is not necessary to establish a perfectly controlled SOP and a universally available common cut-off value. If blood samples of a prescreening population are prepared under the same pre-analytic SOP (blood sampling, processing and storage), what we need to do is sort the individuals according to measured plasma biomarker values, and then select individuals from the top according to the expected prevalence of $A \beta$-positivity in the population. This could be a kind of "consider while running" style, meaning that while trying prescreening applications, we can accumulate data necessary for further validation, determining ideal SOP and common cut-off values that help to establish general clinical utility of the plasma biomarkers. To start this challenge, scalability and global applicability of the measurement system will be required.
Researchers in Shimadzu have already developed a semi-automatic IP-MS system that enables assay of about 100 samples/day under stably-controlled analytic factors. They are also planning to expand the system internationally. If this initial application is successful, it should be very helpful in facilitating drug trials.

\section{References}

1. Henriksen K, O’Bryant SE, Hampel H, Trojanowski JQ, Montine TJ, Jeromin A, et al. The future of blood-based biomarkers for Alzheimer's disease. Alzheimers Dement. 2014;10(1):115-31.

2. O'Bryant SE, Mielke MM, Rissman RA, Lista S, Vanderstichele H, Zetterberg $\mathrm{H}$, et al. Blood-based biomarkers in Alzheimer disease: Current state of the science and a novel collaborative paradigm for advancing from discovery to clinic. Alzheimers Dement. 2016.

3. Wood H. Alzheimer disease: Biomarkers of AD risk - the end of the road for plasma amyloid-beta? Nat Rev Neurol. 2016;12(11):613.

4. Fandos N, Perez-Grijalba V, Pesini P, Olmos S, Bossa M, Villemagne VL, et al. Plasma amyloid beta 42/40 ratios as biomarkers for amyloid beta cerebral deposition in cognitively normal individuals. Alzheimer's \& dementia (Amsterdam, Netherlands). 2017;8:179-87.

5. Ovod V, Ramsey KN, Mawuenyega KG, Bollinger JG, Hicks T, Schneider T, et al. Amyloid beta concentrations and stable isotope labeling kinetics of human plasma specific to central nervous system amyloidosis. Alzheimers Dement. 2017;13(8):841-9.

6. Nabers A, Perna L, Lange J, Mons U, Schartner J, Güldenhaupt J, et al. Amyloid blood biomarker detects Alzheimer's disease. EMBO Mol Med. 2018.

7. Nakamura A, Kaneko N, Villemagne VL, Kato T, Doecke J, Dore V, et al. High performance plasma amyloid- $\beta$ biomarkers for Alzheimer's disease. Nature. 2018;554(7691):249-54.

8. Kaneko N, Nakamura A, Washimi Y, Kato T, Sakurai T, Arahata Y, et al. Novel plasma biomarker surrogating cerebral amyloid deposition. Proc Jpn Acad Ser B Phys Biol Sci. 2014;90(9):353-64.

9. O'Bryant SE, Gupta V, Henriksen K, Edwards M, Jeromin A, Lista S, et al. Guidelines for the standardization of preanalytic variables for blood-based biomarker studies in Alzheimer's disease research. Alzheimers Dement. 2015;11(5):549-60

10. Rowe CC, Ellis KA, Rimajova M, Bourgeat P, Pike KE, Jones G, et al. Amyloid imaging results from the Australian Imaging, Biomarkers and Lifestyle (AIBL) study of aging. Neurobiol Aging. 2010;31(8):1275-83. 